molecular biology of cellular responses to cytokine stimulation. An investigation of the implications of interleukin- 6 levels in the amniotic fluid of pregnant women at parturition provides a good example of a novel report from the clinical front line. In addition to the full papers presenting complete pieces of work, a positive feature of Cytokine is the inclusion of brief reports of less than four pages presenting novel findings in a succinct fashion. Most of the papers are of good quality, being well written and informative. The policy of having occasional reviews is a good one, as it will allow readers to keep up with advances in understanding of the properties of cytokines other than those of their own immediate interest.

The breadth of coverage is in keeping with the editors' stated wish to attract contributions to Cytokine from sources other than immunology and haematology research groups. But a nagging feature of the issues provided for review was that more than one-third of the papers were concerned with interleukin1 in some way; reports dealing with interleukin-6 were also in abundance. Although this might reflect the inherent pleiotropy of these two cytokines, it could signal a trend towards Cytokine becoming a journal aimed largely at those interested in interleukin-1 and -6 , which would be a great pity. I am sure that a more balanced coverage of all the principal cytokines will be achieved as Cytokine becomes better known and recognized by workers in the field as a whole.

The quality of production is very high. In particular, the reproduction of graphical material, tissue sections and autoradiographs is excellent. The bold blue cover of the journal, complete with original abstract painting, is doubtlessly designed to ensure the journal stands out on the library shelf. The challenge for the editors will be to keep up the high quality of papers and to ensure that the contents are as eye-catching as the cover.

William Cushley is in the Department of Biochemistry, University of Glasgow, Glas gow G12 8QQ, Scotland, UK.

\title{
Something for all families
}

Robin A. Weiss

Seminars in Virology. Guest editors. Saunders. 6/yr. US $\$ 120$, e/sewhere $\$ 145$ (institutional); US \$64, elsewhere $\$ 85$ (industrial); US $\$ 48$, elsewhere $\$ 69$ (personal).

SEMINARS in Virology is a recent addition to the Saunders series, commencing publication in January 1990. Each issue comprises $6-8$ short reviews on related topics coordinated by a distinguished guest editor chosen for the particular field. An attractive feature is that most issues treat an aspect of virology that spans different virus families. Thus the first issue dealt with modern approaches to vaccines (editor, F. Brown), the fourth with mechanisms of viral pathogenicity, including plant and insect viruses (editor, R. S. Fujinami), and the sixth with virus structure (editor, J. Hogle). Some issues cover a particular

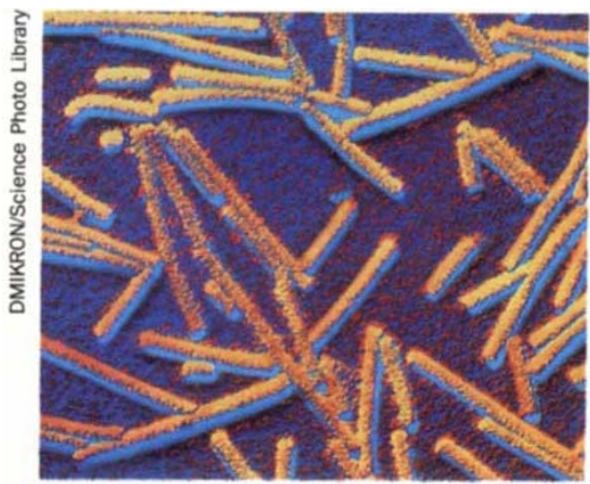

Tobacco mosaic virus $(\times 72,600)$, the cause of leaf mosaic disease in tobacco plants. virus group, but even then there is breadth of treatment.

The individual review articles cover the authors' special interests, and the choice of chapters in each issue does not attempt to be comprehensive. This adds spice to the articles, as they are written by enthusiasts. In each issue I found several interesting and illuminating reviews. By and large, the articles are well illustrated and successfully encapsulate current knowledge and ideas in their respective fields.

There are few other review journals in virology and this one is a welcome addition to the field. Whether the topics and treatment will remain as lively and tidy as they were in the first nine issues remains to be seen, but I can recommend the series to virologists from graduate students onwards. It is doubtful whether the journal as a series will attract much interest among nonvirologists, but individual issues should. Viruses have been at the forefront of numerous basic discoveries in cell and molecular biology and deserve attention beyond the field of infection.

Robin A. Weiss is in the Chester Beatty Laboratories, The Institute of Cancer Research, Fulham Road, London SW3 6JB, UK.

\section{Special agents}

\section{T.A. Connors}

Antl-Cancer Drugs: An International Journal on Anti-Cancer Agents. Editor Mels Sluyser. Rapid Communications of Oxford. 6/yr. £222, \$399.

THE ultimate aim of every cancer chemotherapist is to design the perfect anticancer agent - a chemical that will cure patients of a cancer at a dose that has no side-effects. It was rather surprising, therefore, to read in the aims and scope of the journal that "the editors recognised the need to encourage research on non-toxic cancer agents". Assuming they mean anticancer agents, then chemotherapists have been attempting to do this for years and are hardly in need of encouragement. Toxicity is not deliberately designed into the molecule but is rather the sad consequence of being unable to discover truly selective anticancer agents. Reading further, it appears that the editor is distinguishing between cytotoxic agents and biologicalresponse modifiers, the former being classed as toxic and the latter as nontoxic. Whether biological-response modifiers can be considered to be a nontoxic class of agents is arguable, but one nevertheless follows the line of thinking.

All things considered, the journal has got off to a good start, no doubt assisted by its rapid publication policy which aims to peer review and publish acceptable papers within 30-60 days. Wisely, and presumably to guard against a possibly low level of submission of original papers in the early editions, a number of review papers have been commissioned. These are wide-ranging and uniformly good, some such as Judson's on anthrapyrazoles being very topical.

In the editor's own words, the research papers comprise a plethora of subjects, which is a little unkind, since plethora is usually defined as an overfullness or unhealthy excess. Certainly the subjects covered are wide-ranging and deal with anticancer drugs in the broadest possible sense. It is to be hoped that some of the 'research' articles that are case reports of toxicity will not feature prominently in the future, nor articles that describe the effects of nitrogen mustards on transplanted tumours, bearing in mind that by 1960 more than 2,000 nitrogen mustards had been synthesized and shown to be active against transplanted tumours. I assume that the unimaginative papers will eventually be replaced by more original articles, of which there is already some evidence.

The rapid review system appears to be working well, with all published papers 